\title{
Codimension Two Compact Hausdorff Foliations by Hyperbolic Surfaces Are Not Stable
}

\author{
By \\ Kazuhiko FUKUI* and Atsuhide MORI**
}

\section{$\S 1 . \quad$ Introduction}

The set of codimension $q C^{r}$-foliations of a $C^{r}-n$-manifold $M$ carries the fine $C^{r}$-topology described in [6]. We denote this space by $F_{0} l_{q}^{r}(M)$. We say a foliation $\mathcal{F}$ is $C^{r}$-stable if there is a neighborhood $V$ of $\mathcal{F}$ in $F o l_{q}^{r}(M)$ such that any foliation in $V$ has a compact leaf. $\mathcal{F}$ is $C^{r}$-unstable if not. A foliation in a small neighborhood of $\mathcal{F}$ in $\operatorname{Fol}_{q}^{r}(M)$ is said to be a $C^{r}$-perturbation of $\mathcal{F}$.

There are many works about the stability of foliations. Let $L$ be a compact leaf of $\mathcal{F}$. Langevin-Rosenberg[12] showed, generalizing the Reeb stability theorem [15], that $\mathcal{F}$ is $C^{1}$-stable if $H^{1}(L ; \mathbf{R})=0$. Let $\pi_{1}(L) \rightarrow G L(q, \mathbf{R})$ be the action determined from the linear holonomy of $L$. Stowe[18] showed, generalizing the results of Hirsch[11] and Thurston[19], that $\mathcal{F}$ is $C^{1}$-stable if the cohomology group $H^{1}\left(\pi_{1}(L) ; \mathbf{R}^{q}\right)$ is trivial.

On the other hand, as for the foliation $\mathcal{F}$ of an orientable $S^{1}$-bundle over a closed surface $B$ by fibres, Seifert[17] showed that $\mathcal{F}$ is $C^{0}$-stable if $\chi(B) \neq 0$, where $\chi(B)$ is the euler characteristic of $B$. This result was generalized by Fuller[10] and Fukui[8] : Fuller generalized this to orientable $S^{1}$ bundles over arbitrary closed manifolds with $\chi(B) \neq 0$, and Fukui classified all codimension two compact $C^{r}$-foliations of closed $C^{r}$-3-manifolds $(r \geq 1)$ into $C^{1}$-stable or $C^{r}$-unstable ones. Langevin-Rosenberg[13] considered a fibration $\pi: M \rightarrow B$ with fibre $L$ and showed that the foliation by fibres is $C^{0}$-stable if 1) $\left.\pi_{1}(L) \cong \mathbf{Z}, 2\right) B$ is a closed surface with $\chi(B) \neq 0$, and 3) $\pi_{1}(B)$ acts trivially on $\pi_{1}(L)$. Fukui[6] generalized this result to compact codimension

\footnotetext{
Communicated by K. Saito, May 25, 1998. Revised July 12, 1999.

1991 Mathematics Subject Classification(s): 57R30.

*This research was partially supported by Grant-in Aid for Scientific Research (No. 10640096), Ministry of Education, Science and Culture, Japan.

*Department of Mathematics, Kyoto Sangyo University, Kyoto 603-8555, Japan. e-mail: fukui@cc.kyoto-su.ac.jp

** Department of Mathematics, Kyoto Sangyo University, Kyoto 603-8555, Japan. e-mail: rimo@cc.kyoto-su.ac.jp
} 
two foliations. Plante[14] classified all transversely orientable $C^{r}$-foliations of closed $C^{r}$-3-manifolds by closed orientable surfaces $(r \geq 1)$ into $C^{1}$-stable or $C^{r}$-unstable ones. Especially, foliations by hyperbolic surfaces are $C^{r}$-unstable, where a closed orientable surface is said to be elliptic, parabolic, or hyperbolic if it is of genus 0,1 , or $\geq 2$ respectively. Morover, Tsuboi[20] showed, improving the result of Bonatti-Firmo[2], that a transversely orientable foliation $\mathcal{F}\left(\in \mathrm{Fol}_{1}^{2}(M)\right)$ of a 3 -manifold $M$ is $C^{1}$-unstable if all compact leaves of $\mathcal{F}$ are hyperbolic. As for the stability of foliations of closed 4-manifolds by closed orientable surfaces, we can summarize as follows (Bonatti[1],Fukui[9] et al):

A) The case where $\mathcal{F}\left(\in \operatorname{Fol}_{2}^{1}(M)\right)$ is a foliation by elliptic surfaces $S^{2}$ :

In this case the Reeb stability theorem implies that $\mathcal{F}$ is $C^{1}$-stable.

B) The case where $\mathcal{F}\left(\in \operatorname{Fol}_{2}^{1}(M)\right)$ is a foliation by parabolic surfaces $T^{2}$ :

1) The product foliation $\mathcal{F}$ of $M=T^{2} \times B$ is $C^{1}$-stable if and only if $\chi(B) \neq 0$.

2) $\mathcal{F}$ is $C^{1}$-stable if $\mathcal{F}$ has a rotation or a dihedral leaf.

3) $\mathcal{F}\left(\in F_{o l}^{2}(M)\right)$ is $C^{2}$-stable if $\mathcal{F}$ has reflection leaves with

$$
h_{*}=\left(\begin{array}{cc}
-1 & 0 \\
0 & -1
\end{array}\right)
$$

where $h_{*}: H_{1}\left(T^{2} ; \mathbb{Z}\right) \rightarrow H_{1}\left(T^{2} ; \mathbb{Z}\right)$ is the automorphism induced from the attaching diffeomorphism $h: T^{2} \rightarrow T^{2}$ and we regard the connected components of the reflection leaves as $T^{2} \times[0,1] / h$.

C) The case where $\mathcal{F}\left(\in \operatorname{Fol}_{2}^{1}(M)\right)$ is a foliation by hyperbolic surfaces:

In this case $\mathcal{F}$ is $C^{r}$-unstable if the genus of the generic leaf is sufficiently large.

We study here the case of $\mathrm{C}$ ). Our main result is the following.

Theorem (Theorem 11). All compact Hausdorff $C^{r}$-foliations of $C^{r}$-4manifolds by hyperbolic surfaces are $C^{r}$-unstable $(1 \leq r \leq \infty)$.

Note that $M$ need not be compact, but if it is compact, then by the results of Epstein[4], Edwards, Millett and Sullivan[3], codimension two compact foliations of compact manifolds are always Hausdorff ones. Thus our result means that $\mathcal{F}$ is always $C^{r}$-unstable in the case of $\mathrm{C}$ ).

The authers would like to thank the referee for pointing out a gap in the first version and for helpful suggestions. Especially, the referee suggested the argument in $\S 3$ taking local sections to prove the non-existence of compact leaves, which made the explanations more precise. 


\section{§2. Compact Hausdorff Foliations and Singular Leaves}

Let $M$ be a $C^{r}$-n-manifold without boundary and $\mathcal{F}$ a codimension $q$ compact Hausdorff $C^{r}$-foliation of $M(1 \leq r \leq \infty)$, where $\mathcal{F}$ is said to be Hausdorff if the leaf space $M / \mathcal{F}$ is Hausdoff. Then we have a nice picture of the local behavior of $\mathcal{F}$ as follows.

Proposition 1 (Epstein[5]). There is a generic leaf $L_{0}$ with property that there is an open dense subset of $M$, where the leaves have all trivial holonomy and are all diffeomorphic to $L_{0}$. Given a leaf $L$, we can describe a neighborhood $U(L)$ of $L$, together with the foliation on the neighborhood as follows. There is a finite subgroup $G(L)$ of $O(q)$ such that $G(L)$ acts freely on $L_{0}$ on the right and $L_{0} / G(L) \cong L$. Let $D^{q}$ be the unit disk. We foliate $L_{0} \times D^{q}$ with leaves of the form $L_{0} \times\{p t\}$. This foliation is preserved by the diagonal action of $G(L)$, defined by $g(x, y)=\left(x \cdot g^{-1}, g \cdot y\right)$ for $g \in G(L), x \in L_{0}$ and $y \in D^{q}$ where $G(L)$ acts linearly on $D^{q}$. So we have a foliation induced on $U=L_{0} \times_{G(L)} D^{q}$. The leaf corresponding to $y=0$ is $L_{0} / G(L)$. Then there is a $C^{r}$-imbedding $\varphi: U \rightarrow M$ with $\varphi(U)=U(L)$, which preserves leaves and $\varphi\left(L_{0} / G(L)\right)=L$.

Hereafter we consider the case of codimension 2 in this paper. Since $G(L)$ is a finite subgroup of $O(2)$, the action of $G(L)$ is isomorphic to that of a rotation group $\mathbb{Z}_{k}(k>1)$, a reflection group $\mathbf{D}$ or a dihedral group $\mathbb{D}_{l}(l>1)$.

Definition 2. A leaf $L$ is singular if $G(L)$ is not trivial. The order of $G(L)$ is called the order of holonomy of $L$. We say such an $L$ is a rotation leaf, a reflection leaf or a dihedral leaf if the action of $G(L)$ is isomorphic to that of $\mathbb{Z}_{k}(k>1), \mathbf{D}$ or $\mathbb{D}_{l}(l>1)$ respectively.

\section{$\S 3 . \quad$ Instability of a Foliation by Fibres}

In this section, we consider the stability of bundle foliations. Let $L$ be a hyperbolic surface and $\pi: M \rightarrow B$ a $C^{r}$-fibre bundle with fibre $L$ over a closed surface $B$. Let $\mathcal{F}$ be a foliation of $M$ by fibres and $g(L)$ the genus of the hyperbolic surface $L$. Then we have the following theorem by the similar way as Plante[14] and Fukui[9].

Theorem 3. $\mathcal{F}$ is $C^{r}$-unstable if $g(L) \geq 3(0 \leq r \leq \infty)$.

We can prove this theorem by using the following Remark 4 and Lemma 5.

Remark 4 (Construction of $C^{r}$-perturbed cassette). Let $U$ be an open neighborhood in a $C^{r}$-2-manifold $M$ which is $C^{r}$-diffeomorphic to the open unit disk $i n t D^{2}=\left\{(x, y) \in \mathbf{R}^{2} \mid x^{2}+y^{2}<1\right\}$ via a diffeomorphism $\varphi$. Take a family of diffeomorphisms $\left\{f_{t}: U \rightarrow U \mid t \in\left[\frac{1}{3}, \frac{2}{3}\right]\right\}$ such that 
1) $f_{t}$ is $C^{r}$-close to the identity in the fine $C^{r}$-topology for $t \in\left[\frac{1}{3}, \frac{2}{3}\right]$,

2) $f_{t}=i d$ for $t \in\left[\frac{1}{3}, \frac{4}{9}\right]$,

3) $f_{t}=f_{\frac{2}{3}}$ for $t \in\left[\frac{5}{9}, \frac{2}{3}\right]$ and

4) for fixed $p(\in U),\left(t, f_{t}(p)\right):\left[\frac{1}{3}, \frac{2}{3}\right] \rightarrow\left[\frac{1}{3}, \frac{2}{3}\right] \times U$ is a $C^{r}$-imbedding.

5) $f=f_{\frac{2}{3}}$ has no periodic points.

We say that the $C^{r}$-foliation of $\left[\frac{1}{3}, \frac{2}{3}\right] \times U$ by curves of the above 4 ) is a $C^{r}$-perturbed cassette for the product foliation of $\left[\frac{1}{3}, \frac{2}{3}\right] \times U$ and denote it by $P^{r}(U, f)$. For example, for a function $h_{1}(t)=\exp ((1 / t(t-1))(0<t<1)$, $h_{1}(t)=0(t \leq 0$ or $t \geq 1)$ and small $\epsilon>0$, putting

$$
h_{2}(s)=\int_{0}^{s} h_{1}(t) d t / \int_{0}^{1} h_{1}(t) d t, \quad h(x, y)=\left(x+\epsilon h_{2}\left(2-2 \sqrt{x^{2}+y^{2}}\right), y\right),
$$

we can take a family of diffeomorphisms $\left\{f_{t}: U \rightarrow U ; t \in\left[\frac{1}{3}, \frac{2}{3}\right]\right\}$ defined by

$$
\varphi \circ f_{t} \circ \varphi^{-1}(x, y)=(x, y)+h_{2}(9 t-4)(h(x, y)-(x, y)) \text {. }
$$

Lemma 5. Let $L$ be a closed orientable surface and $g$ the genus of $L$. For given $(2 g-2)$ simple closed curves $\delta_{1}, \delta_{2}, \cdots, \delta_{2 g-2}$ in $L$, there is a simple closed curve $\delta$ such that

$$
[\delta] \neq 0,[\delta] \neq\left[\delta_{1}\right],[\delta] \neq\left[\delta_{1}\right], \cdots,[\delta] \neq\left[\delta_{2 g-2}\right]
$$

and

$$
\left\langle[\delta],\left[\delta_{1}\right]\right\rangle=\left\langle[\delta],\left[\delta_{2}\right]\right\rangle=\cdots=\left\langle[\delta],\left[\delta_{2 g-2}\right]\right\rangle=0,
$$

where [ ] denotes the homology class of a simple closed curve in $L$ and $\langle$, denotes the algebraic intersection number of 1-cycles in $L$.

Proof. Let $\alpha_{i}, \beta_{i}(1 \leq i \leq g)$ be non-null-homologous simple closed curves in $L$ such that

$$
\left\langle\left[\alpha_{i}\right],\left[\alpha_{j}\right]\right\rangle=\left\langle\left[\beta_{i}\right],\left[\beta_{j}\right]\right\rangle=0,\left\langle\left[\alpha_{i}\right],\left[\beta_{j}\right]\right\rangle=\delta_{i j}(\forall i, j) .
$$

Then $\left[\alpha_{i}\right]$ and $\left[\beta_{i}\right](1 \leq i \leq g)$ form a canonical symplectic basis for $H_{1}(L ; \mathbb{Z})$. The homology class of $\delta_{j}$ is expressed as $\left[\delta_{j}\right]=\sum_{i=1}^{g}\left(k_{i j}\left[\alpha_{i}\right]+l_{i j}\left[\beta_{i}\right]\right)(j=$ $1,2, \cdots, 2 g-2)$. Put $[\delta]=\sum_{i=1}^{g}\left(k_{i}\left[\alpha_{i}\right]+l_{i}\left[\beta_{i}\right]\right)$. Then equations $\left\langle[\delta],\left[\delta_{j}\right]\right\rangle=0$ $(j=1,2, \cdots, 2 g-2)$ can be solved with $[\delta] \neq 0,[\delta] \neq\left[\delta_{1}\right],[\delta] \neq\left[\delta_{2}\right], \cdots,[\delta] \neq$ $\left[\delta_{2 g-2}\right]$. Note that $\delta$ can be realized as a simple closed curve in $L$ if $k_{1}, l_{1}, \cdots, k_{g}, l_{g}$ are relatively prime.

In the above proof, $\left[\delta_{1}\right],\left[\delta_{2}\right], \cdots,\left[\delta_{2 g-2}\right]$ are linearly independent when the dimension of the solution space of equations $\left\langle[\delta],\left[\delta_{j}\right]\right\rangle=0(j=1,2, \cdots, 2 g-2)$ 
is two and then $\left[\delta_{1}\right],\left[\delta_{2}\right], \cdots,\left[\delta_{2 g-2}\right]$ are different from each other. Thus, the following corollary holds.

Corollary 6. Let $L$ be a closed orientable surface and $g$ the genus of $L$. For given $(2 g-1)$ simple closed curves $\delta_{1}, \delta_{2}, \cdots, \delta_{2 g-1}$ in $L$, there is a simple closed curve $\delta$ such that 1$) \quad[\delta] \neq 0$,

2) $[\delta]$ is equal to at most one of $\left[\delta_{1}\right],\left[\delta_{2}\right], \cdots,\left[\delta_{2 g-1}\right]$ and

3) $\left\langle[\delta],\left[\delta_{1}\right]\right\rangle=\left\langle[\delta],\left[\delta_{2}\right]\right\rangle=\cdots=\left\langle[\delta],\left[\delta_{2 g-1}\right]\right\rangle=0$.

Now we are in a position to prove Theorem 3.

Proof of Theorem 3. We consider the dual cell decomposition of a small triangulation of the base space $B$ and denote each of these polygons by $P_{i}(i \in$ $I)$. Let $V_{i}\left(\supset P_{i}\right)$ be a neighborhood diffeomorphic to an open disk $(i \in I)$. We can assume that $\pi$ is trivial over each $V_{i}$ and let $\psi_{i}: \pi^{-1}\left(V_{i}\right) \rightarrow L \times V_{i}$ be a trivialization of $\pi$ over $V_{i}(i \in I)$. Put $\psi_{j i}=\psi_{j} \circ \psi_{i}^{-1}$. Let $\delta_{i}$ be a simple closed curve in $L$ of $L \times V_{i}$, then $\psi_{j i}\left(\delta_{i} \times\{p\}\right)\left(p \in V_{i} \cap V_{j}\right)$ is homologous, in each fibre, to some simple closed curve $\delta_{i}^{\prime}$ in $L$ of $L \times V_{j}$, that is, $\psi_{j i}\left(\left[\delta_{i}\right]\right)=\left[\delta_{i}^{\prime}\right]$.

If $P_{i} \cap P_{j} \cap P_{k}$ is a vertex, take a neighborhood $U_{i j k}$ of the vertex such that $U_{i j k} \subset V_{i} \cap V_{j} \cap V_{k}, U_{i j k}$ is diffeomorphic to an open disk and $U_{i j k} \cap U_{i^{\prime} j^{\prime} k^{\prime}}=\emptyset$ for a neighborhood $U_{i^{\prime} j^{\prime} k^{\prime}}$ of another vertex.

If $P_{i} \cap P_{j}$ is an edge, take a neighborhood $U_{i j}$ of $\left(P_{i} \cap P_{j}\right)-\left(U_{i j k} \cup U_{i j l}\right)$ such that $U_{i j} \subset V_{i} \cap V_{j}, U_{i j}$ is diffeomorphic to an open disk and $U_{i j} \cap U_{i^{\prime} j^{\prime}}=\emptyset$ for a neighborhood $U_{i^{\prime} j^{\prime}}$ of another (shortened) edge, where $U_{i j k}$ and $U_{i j l}$ are the neighborhoods of the ends of the edge.

We can take an open set $U_{i}\left(\subset P_{i}\right)$ diffeomorphic to an open disk for each $i \in I$ such that $\left\{U_{i} ; i \in I\right\} \cup\left\{U_{i j k} ; P_{i} \cap P_{j} \cap P_{k}\right.$ is a vertex $\} \cup\left\{U_{i j} ; P_{i} \cap P_{j}\right.$ is an edge $\}$ is an open covering of the base space $B$ as shown in Fig.1(a).

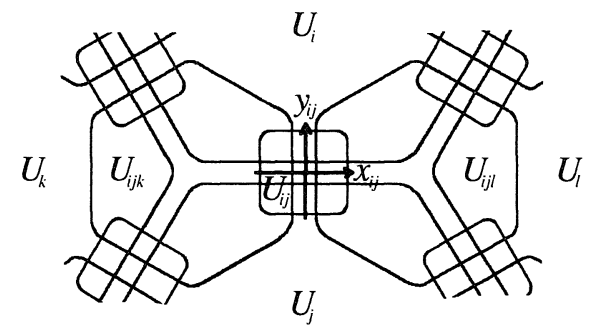

Fig. 1(a).

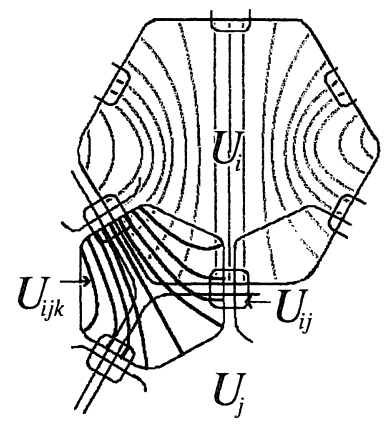

Fig. 1(b).

Take a local coodinate $\left(x_{i j}, y_{i j}\right)$ on each $U_{i j}$ such that $\partial U_{i}$ and $\partial U_{j}$ (resp. $\partial U_{i j k}$ and $\partial U_{i j l}$ ) are two lines parallel to the $x_{i j}$ axis (resp. $y_{i j}$ axis) in $U_{i j}$. 
Step 1. We perturb $\mathcal{F}$ on $L \times U_{i}$ as follows. Let $\gamma_{i}$ be a non-nullhomologous simple closed curve in $L$ of $L \times U_{i}$ and $Q$ an open tubular neighborhood of $\gamma_{i}$ in $L$ such that its closure $\bar{Q}$ is homeomorphic to $\gamma_{i} \times[0,1]$ with coordinate $(s, t), s \in \gamma_{i}, t \in[0,1]$. Since the holonomy of $L$ in $\mathcal{F}$ along $\gamma_{i}$ is trivial, the foliation induced on $\bar{Q} \times U_{i}$ has leaves of form $\bar{Q} \times\{y\}$. In the restriction of $\mathcal{F}$ to $\gamma_{i} \times[0,1] \times U_{i}$, we change the part $\gamma_{i} \times\left[\frac{1}{3}, \frac{2}{3}\right] \times U_{i}$ to $\gamma_{i} \times P^{r}\left(U_{i}, f_{i}\right)$, where $P^{r}\left(U_{i}, f_{i}\right)$ is a $C^{r}$-perturbed cassette in Remark 4 . By such a $C^{r}$-perturbation for each $i$, we obtain a new foliation $\mathcal{F}^{\prime}$ which has no compact leaves in each $\pi^{-1}\left(U_{i}\right)$. We can choose each $\left(\gamma_{i}, f_{i}\right)$ such that $\left\langle\left[\gamma_{i}\right], \psi_{i j}\left(\left[\gamma_{j}\right]\right)\right\rangle \neq 0$ and $f_{i}$ coincides with the time- 1 map determined by a vector field of the form $\lambda_{i j}\left(y_{i j}\right) \frac{\partial}{\partial y_{i j}}$ on $U_{i} \cap U_{i j}$ for any pair $(i, j)$ defining $U_{i j}$ (see Fig.1(b)).

Step 2. We perturb $\mathcal{F}^{\prime}$ on $\pi^{-1}\left(U_{i j k}\right)$ as follows. Before the perturbation of the above step 1, take a simple closed curve $\gamma_{i j k}$ in $L$ of $L \times U_{i j k}\left(\subset L \times V_{i}\right)$ which satisfies

$$
\left[\gamma_{i j k}\right] \neq 0,\left[\gamma_{i j k}\right] \neq\left[\gamma_{i}\right],\left[\gamma_{i j k}\right] \neq \psi_{i j}\left(\left[\gamma_{j}\right]\right),\left[\gamma_{i j k}\right] \neq \psi_{i k}\left(\left[\gamma_{k}\right]\right)
$$

and

$$
\left\langle\left[\gamma_{i j k}\right],\left[\gamma_{i}\right]\right\rangle=\left\langle\left[\gamma_{i j k}\right], \psi_{i j}\left(\left[\gamma_{j}\right]\right)\right\rangle=\left\langle\left[\gamma_{i j k}\right], \psi_{i k}\left(\left[\gamma_{k}\right]\right)\right\rangle=0
$$

This is possible by Lemma 5 . Since the holonomy of $\mathcal{F}^{\prime}$ (after the first perturbation) along $\gamma_{i j k}$ is still trivial, we obtain, by a similar perturbation along this $\gamma_{i j k}$ using $P^{r}\left(U_{i j k}, f_{i j k}\right)$ as in Step 1, a new foliation $\mathcal{F}^{\prime \prime}$.

In order to show that $\mathcal{F}^{\prime \prime}$ has no compact leaves in each $\pi^{-1}\left(U_{i j k}\right)$ nor in each $\pi^{-1}\left(U_{i}\right)$, we take a point $p_{i}$ on $\gamma_{i}$ (resp. $p_{i j k}$ on $\gamma_{i j k}$ ) and a local section $s_{i}: U_{i} \rightarrow M$ (resp. $s_{i j k}: U_{i j k} \rightarrow M$ ) which satisfies $s_{i}\left(U_{i}\right)=\left\{p_{i}\right\} \times\{0\} \times U_{i}$ (resp. $\left.s_{i j k}\left(U_{i j k}\right)=\left\{p_{i j k}\right\} \times\{0\} \times U_{i j k}\right)$. We may assume that each local section does not intersect with the interior of $\left(\gamma_{i} \times[0,1] \times U_{i}\right) \cup\left(\gamma_{i j k} \times[0,1] \times U_{i j k}\right)$. Then any leaf of $\mathcal{F}^{\prime}$ intersecting with $s_{i}\left(U_{i}\right)$ is not compact; To see this, take a closed curve $c: \mathbb{R} \rightarrow \mathbb{R} / \mathbb{Z} \rightarrow M$ which has the geometric intersection number +1 (or -1) with $\gamma_{i}$ on a leaf of $\mathcal{F}$ intersecting with $s_{i}\left(U_{i}\right)$ and consider the lift of $c$ to the corresponding leaf curve on $\mathcal{F}^{\prime}$, then we see that the leaf curve accumulates to a point of $s_{i}\left(\partial U_{i}\right)$. Next we take the lift of $c$ to the corresponding leaf curve on $\mathcal{F}^{\prime \prime}$. If the leaf containing the lift does not intersect with $s_{i j k}\left(U_{i j k}\right)$, then the lift accumulates to a point of $s_{i}\left(\partial U_{i}\right)$. Thus any leaf of $\mathcal{F}^{\prime \prime}$ which does not intersect with $s_{i j k}\left(U_{i j k}\right)$ but intersects with $s_{i}\left(U_{i}\right)$ is not compact. We can also show that any leaf of $\mathcal{F}^{\prime \prime}$ intersecting with $s_{i j k}\left(U_{i j k}\right)$ is not compact as follows; Take a closed curve $c^{\prime}: \mathbb{R} \rightarrow \mathbb{R} / \mathbb{Z} \rightarrow M$ on a leaf of $\mathcal{F}^{\prime}$ which has the geometric intersection number +1 (or -1 ) with one of the components of the lift of $\gamma_{i j k}$ to the corresponding leaf curve on $\mathcal{F}^{\prime}$ and does not intersect with the other (possibly empty) components, then the lift of $c^{\prime}$ to the corresponding leaf curve on $\mathcal{F}^{\prime \prime}$ accumulates to a point of $s_{i j k}\left(\partial U_{i j k}\right)$. We can always take 
the above $c^{\prime}$ since the lift of $\gamma_{i j k}$ does not separate the leaf of $\mathcal{F}^{\prime}$. Thus $\mathcal{F}^{\prime \prime}$ has no compact leaves in each $\pi^{-1}\left(U_{i j k}\right)$ nor in each $\pi^{-1}\left(U_{i}\right)$.

Since the rank of the solution space of the above equations $(*)$ is greater than one, we can choose each $\left(\gamma_{i j k}, f_{i j k}\right)$ such that $\left\langle\left[\gamma_{i j k}\right],\left[\gamma_{i j l}\right]\right\rangle \neq 0$ for $l(\neq k)$ defining $U_{i j l}$ and $f_{i j k}$ coincides with the time-1 map determined by a vector field of the form $\mu_{i j k}\left(x_{i j}\right) \frac{\partial}{\partial x_{i j}}$ on $U_{i j k} \cap U_{i j}$ (see Fig.1(b)).

Step 3. Similarly as above, we can perturb $\mathcal{F}^{\prime \prime}$ by using Lemma 5 . Before the perturbations in the above two steps, take a simple closed curve $\gamma_{i j}$ in $L$ of $L \times U_{i j}\left(\subset L \times V_{i}\right)$ which satisfies

$$
\left[\gamma_{i j}\right] \neq 0,\left[\gamma_{i j}\right] \neq\left[\gamma_{i}\right],\left[\gamma_{i j}\right] \neq \psi_{i j}\left(\left[\gamma_{j}\right]\right),\left[\gamma_{i j}\right] \neq\left[\gamma_{i j k}\right],\left[\gamma_{i j}\right] \neq\left[\gamma_{i j l}\right]
$$

and

$$
\left\langle\left[\gamma_{i j}\right],\left[\gamma_{i}\right]\right\rangle=\left\langle\left[\gamma_{i j}\right], \psi_{i j}\left(\left[\gamma_{j}\right]\right)\right\rangle=\left\langle\left[\gamma_{i j}\right],\left[\gamma_{i j k}\right]\right\rangle=\left\langle\left[\gamma_{i j}\right],\left[\gamma_{i j l}\right]\right\rangle=0
$$

for any pair $(k, l)$ defining $U_{i j k}$ and $U_{i j l}(k \neq l)$. Then, automatically $\left[\gamma_{i}\right],\left[\gamma_{i j k}\right]$ and $\left[\gamma_{i j}\right]$ are linearly independent. By the choice of $f_{i}$ on $U_{i} \cap U_{i j}$ in Step 1 and $f_{i j k}$ on $U_{i j} \cap U_{i j k}$ in Step 2, we may assume that $f_{i}$ and $f_{i j k}$ are commutative on the intersection of their domains in $U_{i j}$ if the length of a sequence consisting of $f_{i}, f_{i j k}$ and their inverses is less than or equal to the geometric intersection number of $\gamma_{i j}$ with $\gamma_{i}$ and $\gamma_{i j k}$. This ensures that the holonomy of $\mathcal{F}^{\prime \prime}$ along $\gamma_{i j}$ is trivial. Thus we obtain, by the perturbation along this $\gamma_{i j}$, a new foliation $\mathcal{F}^{\prime \prime \prime}$ which has no compact leaves in each $\pi^{-1}\left(U_{i j}\right)$.

In order to show that $\mathcal{F}^{\prime \prime \prime}$ has no compact leaves in $M$, we take a point $p_{i j}$ on $\gamma_{i j}$ and a local section $s_{i j}: U_{i j} \rightarrow M$ which satisfies $s_{i j}\left(U_{i j}\right)=\left\{p_{i j}\right\} \times$ $\{0\} \times U_{i j}$. We may assume that each local section does not intersect with the interior of $\left(\gamma_{i} \times[0,1] \times U_{i}\right) \cup\left(\gamma_{i j k} \times[0,1] \times U_{i j k}\right) \cup\left(\gamma_{i j} \times[0,1] \times U_{i j}\right)$. Then any leaf of $\mathcal{F}^{\prime \prime \prime}$ intersecting with $s_{i j}\left(U_{i j}\right)$ is not compact; To see this, take a closed curve $c^{\prime \prime}: \mathbf{R} \rightarrow \mathbf{R} / \mathbb{Z} \rightarrow M$ on a leaf of $\mathcal{F}^{\prime \prime}$ which has the geometric intersection number +1 (or -1 ) with one of the components of the lift of $\gamma_{i j}$ to the corresponding leaf curve on $\mathcal{F}^{\prime \prime}$ and does not intersect with the other (possibly empty) components, then the lift of $c^{\prime \prime}$ to the corresponding leaf curve on $\mathcal{F}^{\prime \prime \prime}$ accumulates to a point of $s_{i j}\left(\partial U_{i j}\right)$. We can always take the above $c^{\prime \prime}$ since the lift of $\gamma_{i j}$ does not separate the leaf of $\mathcal{F}^{\prime \prime}$ as a consequence of the linear independence of $\left[\gamma_{i}\right],\left[\gamma_{i j k}\right]$ and $\left[\gamma_{i j}\right]$. Any leaf of $\mathcal{F}^{\prime \prime \prime}$ which does not intersect with $s_{i j}\left(U_{i j}\right)$ but intersects with $s_{i}\left(U_{i}\right)$ or $s_{i j k}\left(U_{i j k}\right)$ can be identified with the corresponding leaf of $\mathcal{F}^{\prime \prime}$. This leaf has been shown not to be compact in Step 2. Thus $\mathcal{F}^{\prime \prime \prime}$ has no compact leaves in $M$. This completes the proof.

Theorem 3 can be extended to the case of $g(L)=2$ as follows.

Theorem 7. $\mathcal{F}$ is $C^{r}$-unstable if $g(L)=2(0 \leq r \leq \infty)$. 
Proof. In the same setting as in the proof of Theorem 3, we choose simple closed curves $\gamma_{i}\left(\left[\gamma_{i}\right] \neq 0\right)$ in $L$ of $L \times U_{i}(i \in I)$ such that

1) $\left\langle\left[\gamma_{i}\right], \psi_{i j}\left(\left[\gamma_{j}\right]\right)\right\rangle \neq 0$ for any pair $(i, j)$ defining $U_{i j}$.

2) $\left[\gamma_{i}\right], \psi_{i j}\left(\left[\gamma_{j}\right]\right), \psi_{i k}\left(\left[\gamma_{k}\right]\right)$ and $\psi_{i l}\left(\left[\gamma_{l}\right]\right)$ are linearly independent for any quartet $(i, j, k, l)$ defining $U_{i j}, U_{i j k}$ and $U_{i j l}(k \neq l)$.

Putting $\left\langle\left[\gamma_{i}\right], \psi_{i j}\left(\left[\gamma_{j}\right]\right)\right\rangle=p,\left\langle\psi_{i j}\left(\left[\gamma_{j}\right]\right), \psi_{i k}\left(\left[\gamma_{k}\right]\right)\right\rangle=q,\left\langle\psi_{i k}\left(\left[\gamma_{k}\right]\right),\left[\gamma_{i}\right]\right\rangle=r$, we determine the solution of equations

$$
\left\langle\left[\gamma_{i j k}\right],\left[\gamma_{i}\right]\right\rangle=\left\langle\left[\gamma_{i j k}\right], \psi_{i j}\left(\left[\gamma_{j}\right]\right)\right\rangle=\left\langle\left[\gamma_{i j k}\right], \psi_{i k}\left(\left[\gamma_{k}\right]\right)\right\rangle=0
$$

by $\left[\gamma_{i j k}\right]$ (or its multiple) $=q\left[\gamma_{i}\right]+r \psi_{i j}\left(\left[\gamma_{j}\right]\right)+p \psi_{i k}\left(\left[\gamma_{k}\right]\right)$. We can perturb $\mathcal{F}$ by Steps 1,2 in the above proof of Theorem 3 and obtain a new foliation $\mathcal{F}^{\prime \prime}$.

But we cannot take $\gamma_{i j}$ as in the proof of Theorem 3 in this case. So we take a null-homologous simple closed curve $\gamma_{i j}$ representing the homotopy class $\left\{\alpha_{1}\right\}\left\{\beta_{1}\right\}\left\{\alpha_{1}\right\}^{-1}\left\{\beta_{1}\right\}^{-1}$. Then $\gamma_{i j}$ separates each leaf of $\mathcal{F}$ into two once punctured tori $T_{1}$ and $T_{2}$. We may assume that $f_{i}, f_{j}, f_{i j k}$ and $f_{i j l}$ are commutative on the intersection of their domains in $U_{i j}$ if the length of a sequence consisting of $f_{i}, f_{j}, f_{i j k}, f_{i j l}$ and their inverses are less than or equal to the geometric intersection number of $\gamma_{i j}$ with $\gamma_{i}, \gamma_{j}, \gamma_{i j k}$ and $\gamma_{i j l}$, thus the holonomy of $\mathcal{F}^{\prime \prime}$ along $\gamma_{i j}$ is still trivial. Let $F_{1}$ (resp. $F_{2}$ ) be a subset of $U_{i j}$ such that $s_{i j}\left(F_{1}\right)$ (resp. $s_{i j}\left(F_{2}\right)$ ) is the common fixed point set of the holonomies on $s_{i j}\left(U_{i j}\right)$ along loops homotopic to $\alpha_{1}$ and $\beta_{1}$ (resp. $\alpha_{2}$ and $\beta_{2}$ ).

i) First, we consider the case where $F_{1}=U_{i j}-\left(U_{i} \cup U_{j}\right)$ and $F_{2}=U_{i j}-$ $\left(U_{i j k} \cup U_{i j l}\right)$. Let $\gamma_{i}^{\prime}$ and $\gamma_{j}^{\prime}$ (resp. $\gamma_{i j k}^{\prime}$ and $\gamma_{i j l}^{\prime}$ ) be simple closed curves in $T_{1}$ (resp. $T_{2}$ ) each of which is homologous to the corresponding one of $\gamma_{i}$ and $\gamma_{j}$ (resp. $\gamma_{i j k}$ and $\gamma_{i j l}$ ). From the commutativity of the holonomies, we see that the foliation $\mathcal{F}^{\prime \prime}$, which has been perturbed along $\gamma_{i}, \gamma_{j}, \gamma_{i j k}$ and $\gamma_{i j l}$, is $C^{r}$ diffeomorphic in a neighborhood $N_{i j}$ of $L \times\left(U_{i j}-\left(U_{i} \cup U_{j} \cup U_{i j k} \cup U_{i j l}\right)\right)$ to the foliation which is obtained from $\mathcal{F}$ by the perturbations along $\gamma_{i}^{\prime}, \gamma_{j}^{\prime}, \gamma_{i j k}^{\prime}$ and $\gamma_{i j l}^{\prime}$ in the same way. We may assume $L \times U_{i j} \subset N_{i j}$ by taking $U_{i j}$ sufficiently small if necessary.

Let $\nu\left(x_{i j}\right), \xi\left(y_{i j}\right)$ and $\eta\left(y_{i j}\right): U_{i j} \rightarrow[0,1]$ be $C^{r}$-functions as shown in Fig.2(a) and $g_{i j}^{i}: U_{i j} \rightarrow U_{i j}$ (resp. $g_{i j}^{j}: U_{i j} \rightarrow U_{i j}$ ) the time-1 map determined by $\left.\eta\left(y_{i j}\right) \nu\left(x_{i j}\right) \xi\left(y_{i j}\right) \frac{\partial}{\partial x_{i j}}\left(\operatorname{resp.}\left(1-\eta\left(y_{i j}\right)\right) \nu\left(x_{i j}\right) \xi\left(y_{i j}\right) \frac{\partial}{\partial x_{i j}}\right)\right)$. Let $S_{i j}$ be the support of the function $\nu\left(x_{i j}\right) \xi\left(y_{i j}\right)$. We define a perturbation of $\mathcal{F}$ as follows: Cut $T_{1}$ of $T_{1} \times U_{i j}$ along $\gamma_{i}^{\prime}$ and $\psi_{i j}\left(\gamma_{j}^{\prime}\right)$ and paste them along $\gamma_{i}^{\prime}$ (resp. $\left.\psi_{i j}\left(\gamma_{j}^{\prime}\right)\right)$ with sliding by $g_{i j}^{i}$ (resp. $g_{i j}^{j}$ ). This is possible since $g_{i j}^{i}$ and $g_{i j}^{j}$ commute. We can regard this perturbation as a modification of Step 1 as follows. Change $f_{i}$ (resp. $f_{j}$ ) into the time- 1 map determined by $\lambda_{i j}\left(y_{i j}\right) \frac{\partial}{\partial y_{i j}}+$ $\eta\left(y_{i j}\right) \nu\left(x_{i j}\right) \xi\left(y_{i j}\right) \frac{\partial}{\partial x_{i j}}\left(\right.$ resp. $\left.\quad-\lambda_{j i}\left(-y_{i j}\right) \frac{\partial}{\partial y_{i j}}+\left(1-\eta\left(y_{i j}\right)\right) \nu\left(x_{i j}\right) \xi\left(y_{i j}\right) \frac{\partial}{\partial x_{i j}}\right)$. Then we can extend the domain of $f_{i}$ (resp. $f_{j}$ ) to $U_{i j}$. Since this modification is different from the original $\mathcal{F}^{\prime \prime}$ only in $N_{i j}$, we can define a new perturbation 

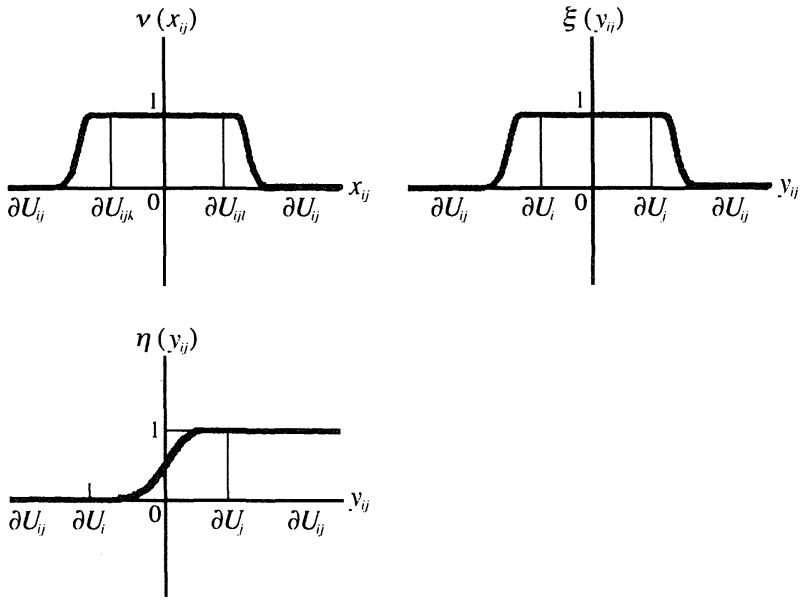

Fig. 2(a).
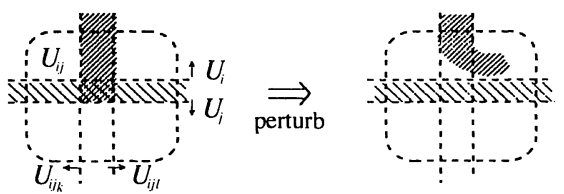

$F_{1}:$ the common fixed point set of the holonomies along $\alpha_{1}$ and $\beta_{1}$
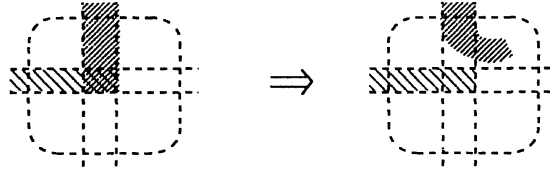

. $I$ III $F_{2}$ : the common fixed point set of the holonomies along $\alpha_{2}$ and $\beta_{2}$
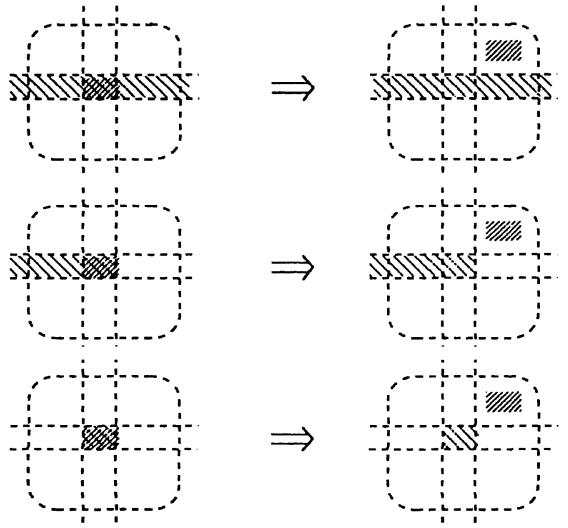

Fig. 2(b). 
$\mathcal{F}_{0}^{\prime \prime \prime}$

Then a leaf of $\mathcal{F}_{0}^{\prime \prime \prime}$ intersecting with $s_{i j}\left(\operatorname{int}\left(S_{i j}\right)-\left(U_{i} \cup U_{j}\right)\right)$ accumulates to a point of $s_{i j}\left(\partial S_{i j}\right)$. Another leaf intersecting with $s_{i j}\left(U_{i j}\right)$ intersects with $s_{i}\left(U_{i}\right), s_{j}\left(U_{j}\right), s_{i j k}\left(U_{i j k}\right)$ or $s_{i j l}\left(U_{i j l}\right)$. By the same argument as in Step 2 in the proof of Theorem 3 , a leaf intersecting with $s_{i}\left(U_{i}\right)$ or $s_{j}\left(U_{j}\right)$ accumulates to a point of $s_{i}\left(\partial U_{i}\right)$ or $s_{j}\left(\partial U_{j}\right)$ since $U_{i}, U_{j}$ are invariant under the new $f_{i}, f_{j}$. In order to show that any leaf which does not intersect with $s_{i j}\left(i n t\left(S_{i j}\right)-\left(U_{i} \cup U_{j}\right)\right)$ but intersects with $s_{i j}\left(U_{i j}\right)$ and $s_{i j k}\left(U_{i j k}\right)$ (resp. $s_{i j l}\left(U_{i j l}\right)$ ) accumulates to a point of $s_{i j k}\left(\partial U_{i j k}\right)\left(\right.$ resp. $\left.s_{i j l}\left(\partial U_{i j l}\right)\right)$, take a closed curve $c: \mathbb{R} \rightarrow \mathbf{R} / \mathbb{Z} \rightarrow M$ on $T_{2}$ in a leaf of $\mathcal{F}^{\prime \prime}$ which has the geometric intersection number +1 (or -1 ) with $\gamma_{i j k}^{\prime}$ (resp. $\gamma_{i j l}^{\prime}$ ), then the lift of $c$ to the corresponding leaf curve on $\mathcal{F}^{\prime \prime}$ accumulates to a point of $s_{i j k}\left(\partial U_{i j k}\right)$.

ii) If we consider the case where $F_{1}=U_{i j}-\left(U_{i j k} \cup U_{i j l}\right)$ and $F_{2}=U_{i j}$ $\left(U_{i} \cup U_{j}\right)$, we conclude the same result as in i).

iii) In the other cases, we may assume that the foliation $\mathcal{F}^{\prime \prime}$ is $C^{r}$-diffeomorphic in a neighborhood of $L \times U_{i j}$ to the foliation which is obtained from $\mathcal{F}$ by the perturbation along $\alpha_{1}, \beta_{1}, \alpha_{2}$ and $\beta_{2}$ by taking $U_{i j}$ sufficiently small if necessary, and let $H\left(\alpha_{m}\right)=f_{i}^{a_{i}^{m}} \circ f_{j}^{a_{j}^{m}} \circ f_{i j k}^{a_{i j k}^{m}} \circ f_{i j l}^{a_{i j l}^{m}}$ (resp. $H\left(\beta_{m}\right)=f_{i}^{b_{i}^{m}} \circ f_{j}^{b_{j}^{m}} \circ f_{i j k}^{b_{i j k}^{m} \circ}$ $\left.f_{i j l}^{b_{i j k}^{m}}\right)$ be the holonomy on $s_{i j}\left(U_{i j}\right)$ along a loop homotopic to $\alpha_{m}$ (resp. $\beta_{m}$ ), where $a_{*}^{m}$ (resp. $b_{*}^{m}$ ) are some integers and we regard each $f_{*}$ as the identity out of the domain, for $m=1,2$. Since $\left[\gamma_{i}\right], \psi_{i j}\left(\left[\gamma_{j}\right]\right),\left[\gamma_{i j k}\right]$ and $\left[\gamma_{i j l}\right]$ are linearly independent, $g(L)=2$ and $\left\langle\left[\gamma_{i}\right],\left[\gamma_{i j k}\right]\right\rangle=\left\langle\left[\gamma_{i}\right],\left[\gamma_{i j l}\right]\right\rangle=\left\langle\psi_{i=j}\left(\left[\gamma_{j}\right]\right),\left[\gamma_{i j k}\right]\right\rangle=$ $\left\langle\psi_{i j}\left(\left[\gamma_{j}\right]\right),\left[\gamma_{i j l}\right]\right\rangle=0$, we see $\left\langle\left[\gamma_{i}\right], \psi_{i j}\left[\gamma_{j}\right]\right\rangle \neq 0,\left\langle\left[\gamma_{i j k}\right],\left[\gamma_{i j l}\right]\right\rangle \neq 0$. Thus either $\left(a_{i}^{1} b_{j}^{1}-a_{j}^{1} b_{i}^{1}\right)\left(a_{i j k}^{2} b_{i j l}^{2}-a_{i j l}^{2} b_{i j k}^{2}\right) \neq 0$ or $\left(a_{i}^{2} b_{j}^{2}-a_{j}^{2} b_{i}^{2}\right)\left(a_{i j k}^{1} b_{i j l}^{1}-a_{i j l}^{1} b_{i j k}^{1}\right) \neq 0$ holds. Then it is sufficient to consider the five cases (up to rotations and reflections) shown in Fig.2(b) by assuming, for example, $\left(a_{i}^{2} b_{j}^{2}-a_{j}^{2} b_{i}^{2}\right)\left(a_{i j k}^{1} b_{i j l}^{1}-a_{i j l}^{1} b_{i j k}^{1}\right) \neq$ 0 . We can always eliminate the intersection of $F_{1}$ and $F_{2}$ by a perturbation using $P^{r}\left(U_{i j}, f_{i j}\right)$ along $\gamma_{i j}$ for example as shown in Fig.2(b) if we take $U_{i j}-\left(U_{i} \cup U_{j} \cup U_{i j k} \cup U_{i j l}\right)$ sufficiently small. We denote this new foliation by $\mathcal{F}_{1}^{\prime \prime \prime}$ and let $F_{1}^{\prime}$ be the image of $F_{1}$ by the perturbation.

A leaf of $\mathcal{F}_{1}^{\prime \prime \prime}$ intersecting with $s_{i j}\left(U_{i j}\right)$ accumulates to a point of $s_{i j}\left(\partial F_{1}^{\prime}\right)$ or $s_{i j}\left(\partial F_{2}\right)$ ); To see this, for example in the fourth case shown in Fig.2(b), we can choose loops $\delta_{1}, \delta_{2}, \ldots, \delta_{8}\left(\right.$ resp. $\left.\delta_{1}^{\prime}, \delta_{2}^{\prime}, \ldots, \delta_{5}^{\prime}\right)$ on each $T_{1}\left(\right.$ resp. $\left.T_{2}\right)$ of $\mathcal{F}$ over 1) $\left.\left.\left.U_{i j k} \cap U_{i}, 2\right) U_{i j k}-\left(U_{i} \cup U_{j}\right), 3\right) U_{i j k} \cap U_{j}, 4\right) U_{j}-\left(U_{i j k} \cup U_{i j l}\right)$, 5) $\left.\left.U_{j} \cap U_{i j l}, 6\right) U_{i j l}-\left(U_{i} \cup U_{j}\right), 7\right) U_{i} \cap U_{i j l}$ and 8) $U_{i}-\left(U_{i j k} \cup U_{i j l}\right)$ (resp. 1) $U_{i}-U_{i j l}$, 2) $U_{i} \cap U_{i j l}$, 3) $U_{i j l}-\left(U_{i} \cup U_{j}\right)$, 4) $U_{i j l} \cap U_{j}$ and 5) $\left.U_{j}-U_{i j l}\right)$ in $U_{i j}$ satisfying that each $\left[\delta_{p}\right]$ (resp. $\left[\delta_{q}^{\prime}\right]$ ) is expressed as $\kappa_{p}\left[\alpha_{1}\right]+\lambda_{p}\left[\beta_{1}\right]$ (resp. $\left.\kappa_{q}^{\prime}\left[\alpha_{2}\right]+\lambda_{q}^{\prime}\left[\beta_{2}\right]\right)$ where $\kappa_{p}$ and $\lambda_{p}$ (resp. $\kappa_{q}^{\prime}$ and $\lambda_{q}^{\prime}$ ) are integers such that $\left(H\left(\alpha_{1}\right)\right)^{\kappa_{p}} \circ\left(H\left(\beta_{1}\right)\right)^{\lambda_{p}}$ (resp. $\left.\left(H\left(\alpha_{2}\right)\right)^{\kappa_{q}^{\prime} \circ}\left(H\left(\beta_{2}\right)\right)^{\lambda_{q}^{\prime}}\right)$ is a contraction to $s_{i j}\left(\partial F_{1}\right)$ (resp. $s_{i j}\left(\partial F_{2}\right)$ ) for $p=1,2, \ldots, 8$ (resp. $\left.q=1,2, \ldots, 5\right)$. Then the lift of each loop to the corresponding leaf curve on $\mathcal{F}^{\prime \prime}$ accumulates to a point 
of $s_{i j}\left(\partial F_{1}\right)$ (resp. $\left.s_{i j}\left(\partial F_{2}\right)\right)$. The other cases can be observed more easily. In any case after the perturbation along $\gamma_{i j}$, a leaf of $\mathcal{F}_{1}^{\prime \prime \prime}$ intersecting $s_{i j}\left(U_{i j}\right)$ accumulates to a point of $s_{i j}\left(\partial F_{1}^{\prime}\right)$ or $s_{i j}\left(\partial F_{2}\right)$, thus this leaf is not compact.

Combining i),ii) and iii), we define a new folliation $\mathcal{F}^{\prime \prime \prime}$ which has no compact leaves in the saturation of each $s_{i j}\left(U_{i j}\right)$. Another leaf can be identified with the corresponding non-compact leaf of $\mathcal{F}^{\prime \prime}$. This completes the proof.

\section{§4. Instability of a Foliation with Singular Leaves}

In this section, we consider the stability of a Hausdorff $C^{r}$-foliation $\mathcal{F}$ of a $C^{r}$-4-manifold by hyperbolic surfaces with singular leaves. We can generalize the above theorems as follows.

A) The case of foliations with only rotation leaves as singular leaves.

Theorem 8. If all the singular leaves of $\mathcal{F}$ are rotation leaves, then $\mathcal{F}$ is $C^{r}$-unstable $(1 \leq r \leq \infty)$.

Proof. In this case, note that the genus of the generic leaves satisfies $g \geq 3$. Let $B=M / \mathcal{F}$ be the leaf space of $\mathcal{F}$. $B$ is a two dimensional $\mathrm{V}$ manifold without boundaries and is also a topological manifold. The quotient map $\pi: M \rightarrow B$ is a V-bundle (see Satake[16] for definitions). We denote the rotation leaves of $\mathcal{F}$ by $L_{j}(j \in J)$. Choose saturated neighborhoods $U\left(L_{j}\right)$ as in Proposition 1 to be disjoint. We denote its interior by $\operatorname{int}\left(U\left(L_{j}\right)\right)$. The map $\pi$ restricted to $M-\cup_{j \in J} L_{j}$ is a fibre bundle with fibre being the generic leaf $L$. So we take a sufficiently fine open covering of $B$ as in the proof of Theorem 3 ,

$$
\left\{U_{i} ; i \in I\right\} \cup\left\{U_{i j k} ; P_{i} \cap P_{j} \cap P_{k} \text { is a vertex }\right\} \cup\left\{U_{i j} ; P_{i} \cap P_{j} \text { is an edge }\right\}
$$

and modify it partially near the points corresponding to rotation leaves as follows (see Fig. 3) :

1) $\quad \operatorname{int}\left(U\left(L_{j}\right)\right)=\pi^{-1}\left(U_{j}\right)(j \in J \subset I)$ and

2) $\quad U_{j} \cap U_{k}=\emptyset(j, k \in J)$.

First we perturb $\mathcal{F}$ on $\operatorname{int}\left(U\left(L_{j}\right)\right)$ as follows. Let $p_{j}: L \rightarrow L_{j}$ be the covering map and $\tau_{j}$ a simple closed curve in $L_{j}$ representing a generator of the holonomy groups $\mathbf{Z}_{k(j)}$ of $L_{j}(j \in J)$. By using Lemma 5 , we can take a simple closed curve $\delta_{j}$ in each $L_{j}$ such that $\left[\delta_{j}\right] \neq 0,\left[\delta_{j}\right] \neq\left[\tau_{j}\right]$ and $\left\langle\left[\tau_{j}\right],\left[\delta_{j}\right]\right\rangle=0$. Since the holonomy of $L_{j}$ along $\delta_{j}$ is trivial, we can perturb $\mathcal{F}$ on $\operatorname{int}\left(U\left(L_{j}\right)\right)$ as in the proof of Theorem 3 and obtain a new foliation $\mathcal{F}^{\prime}$ without compact leaves in each $\operatorname{int}\left(U\left(L_{j}\right)\right)$.

Next we perturb $\mathcal{F}^{\prime}$ on $L \times U_{j i}$ and $L \times U_{j i k}$. Each $\delta_{j}$ induces $k(j)$ simple closed curves $p_{j}^{-1}\left(\delta_{j}\right)=\delta_{j 1} \cup \delta_{j 2} \cup \cdots \cup \delta_{j k(j)}$ in $L$ of $L \times U_{j i}$ and $L \times U_{j i k}$. Since 


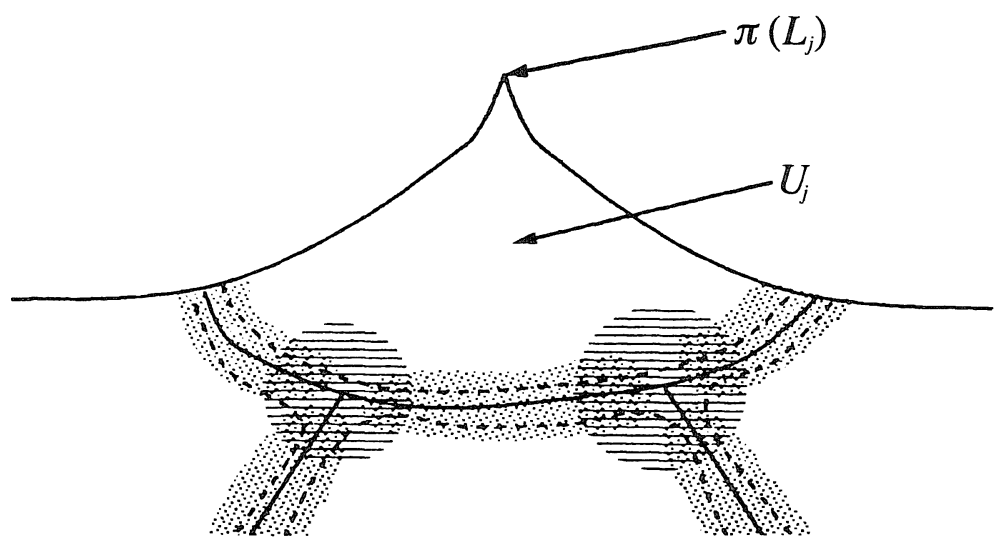

Fig. 3.

the genus of $L \geq k(j)+1$, we can take a simple closed curve $\gamma_{j i}$ on each $U_{j i}$ for the perturbation, by using Corollary 6 and the relation $2 k(j)+1 \geq k(j)+3$ $(k(j) \geq 2)$, where " +3 " means that each $U_{j i}$ meets three other neighborhoods except $U_{j}$. Similarly, we can take a simple closed curve $\gamma_{j i k}$ for the perturbation on each $U_{j i k}$ by Lemma 5 . Since $g \geq 3$, we can perturb $\mathcal{F}^{\prime}$ on the other neighborhoods in $B$ as in the proof of Theorem 3. This completes the proof.

B) The case of foliations with only reflection leaves as singular leaves.

Theorem 9. If all the singular leaves of $\mathcal{F}$ are reflection leaves, then $\mathcal{F}$ is $C^{r}$-unstable $(1 \leq r \leq \infty)$.

Proof. In this case, note that the genus of the generic leaves satisfies $g \geq$ 3. The leaf space $B=M / \mathcal{F}$ is a 2-manifold with boundaries and the quotient map $\pi: M \rightarrow B$ is a $\mathrm{V}$-bundle. Reflection leaves correspond to the boundary points of $B$. Each boundary component of $B$ is homeomorphic to the line $\mathbf{R}$ or the circle $S^{1}$. First we consider the case where $B$ has only one boundary component and denote this component by $\partial B$. Let $L_{x}(x \in \partial B)$ be reflection leaves of $\mathcal{F}$. Choose saturated neighborhoods $U\left(L_{x}\right)$ as in Proposition 1 to be sufficiently small and choose an open covering $\left\{\operatorname{int}\left(U\left(L_{j}\right)\right) ; j \in J=\mathbb{Z} / 2 m\right\}$ ( $m$ is a natural number, but if $\partial B \cong \mathbf{R}$, then $J=\mathbb{Z}, m=+\infty$ ) of $\pi^{-1}(\partial B)$ such that $k \equiv j$ or $j \pm 1 \quad(\bmod 2 m)$ if $\operatorname{int}\left(U\left(L_{k}\right)\right) \cap \operatorname{int}\left(U\left(L_{j}\right)\right) \neq \emptyset$. Since $\pi$ restricted to $M-\pi^{-1}(\partial B)$ is a fibre bundle with fibre $L$, take a sufficiently fine open covering of $B$ as in the proof of Theorem 3,

$$
\left\{U_{i} ; i \in I\right\} \cup\left\{U_{i j k} ; P_{i} \cap P_{j} \cap P_{k} \text { is a vertex }\right\} \cup\left\{U_{i j} ; P_{i} \cap P_{j} \text { is an edge }\right\}
$$

and modify it partially near the boundary as follows (Fig. 4) : 
1) $\quad \operatorname{int}\left(U\left(L_{j}\right)\right)=\pi^{-1}\left(U_{j}\right)(j \in J \subset I)$,

2) If $U_{i}(i \in I-J)$ meets $U_{j} \cap U_{k^{\prime}}\left(j \in J, k^{\prime} \equiv j+1 \quad(\bmod 2 m)\right)$, we denote such $i$ by $i(j)$,

3) $U_{j i(j) i(k)}$ is a sufficiently small neighborhood which meets $U_{j}, U_{i(j)}$ and $U_{i(k)}$, and $U_{i(j) i(k)}$ meets $U_{i(k)}, U_{i(j)}, U_{j i(j) i(k)}$ and $U_{i(j) i(k) l}(j \in J, k \equiv$ $j-1(\bmod 2 m))$ and

4) $\pi$ restricted to the intersection of $U_{j}$ and the neighborhoods which appear in the above 2) and 3) $(j \in J)$ is also a fibre bundle and we can define the transformation map $\psi_{* *}$ over there as in the proof of Theorem 3.

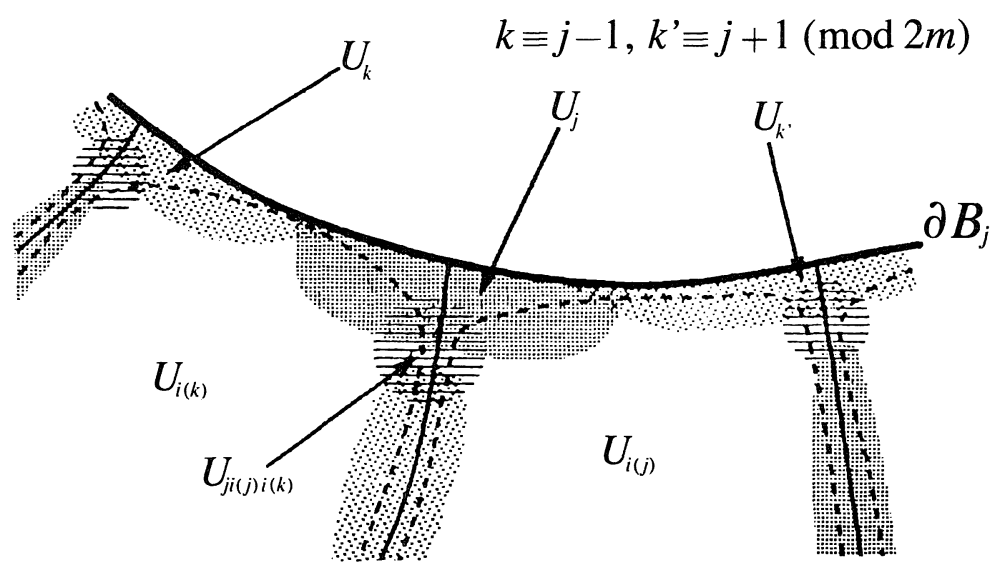

Fig. 4.

Let $\tau_{j}$ be a simple closed curve in $L_{j}$ representing a generator of the holonomy group $\mathbf{D}$ of $L_{j}(j \in J)$.

Step 1 . In the case where $j$ is odd, take an arbitrary simple closed curve $\delta_{j}$ on $L_{j}$ such that $\left[\delta_{j}\right] \neq 0,\left[\delta_{j}\right] \neq\left[\tau_{j}\right]$ and $\left\langle\left[\tau_{j}\right],\left[\delta_{j}\right]\right\rangle=0$ hold and take a tubular neighborhood $S_{j}$ homeomorphic to $\delta_{j} \times(0,1)$. Since the holonomy along $\delta_{j}$ is trivial, $\left.U\left(L_{j}\right)\right|_{S_{j}}$ can be regarded as $S_{j} \times D$, where $D$ is the unit 2-disk. So, we can perturb $\mathcal{F}$ to a foliation which has no compact leaves on $\operatorname{int}\left(U\left(L_{j}\right)\right)$ as in the proof of Theorem 3 .

Step 2. In the case where $j$ is even, we choose a coordinate $(x, y)$ of the unit disk $D^{2}$ in Proposition 1 such that the linear action is given by $g \cdot(x, y)=$ $(x,-y)$. We apply the diffeomorphism $h$ in Remark 4 in the interior of the disk $D^{2}$.

Then, $g \cdot h(x, y)=h(g \cdot(x, y))$ holds. So we obtain a $C^{r}$-perturbation of the foliation of the form $L \times\{p t\}$ by using $h$ which is still preserved by the diagonal action in Proposition 1. This induces a $C^{r}$-perturbation of $\mathcal{F}$ which has no compact leaves on $\operatorname{int}\left(U\left(L_{j}\right)\right)$.

We use a simple closed curve with trivial holonomy in Step 1. On the other 
hand, we use a simple closed curve with non-trivial holonomy in Step 2. Therefore we obtain a new foliation which has no compact leaves on $\cup_{j \in J} \operatorname{int}\left(U\left(L_{j}\right)\right)$ after Steps 1 and 2. We have to note that on neighborhoods near $\partial B$, at most 4 simple closed curves are induced from each $\delta_{j}$ in each reflection leaves $L_{j}$ since $g \geq 3$, but we can perturb on the other neighborhoods as in the proof of Theorem 3 .

We can prove similarly in the case where $B$ has several boundary components. This completes the proof.

C) The case of foliations with dihedral leaves.

Theorem 10. If $\mathcal{F}$ has no rotation leaves, but has dihedral leaves, then $\mathcal{F}$ is $C^{r}$-unstable $(1 \leq r \leq \infty)$.

Proof. The leaf space $B=M / \mathcal{F}$ is a $2-\mathrm{V}$-manifold with boundaries and the quotient map $\pi: M \rightarrow B$ is a $\mathrm{V}$-bundle. Reflection leaves and dihedral leaves correspond to the boundary points of $B$. Now, we may assume that $B$ has only one boundary component $\partial B$ and it is homeomorphic to the line $\mathbf{R}$ or the circle $S^{1}$. Let $L_{x}(x \in \partial B)$ be singular leaves of $\mathcal{F}$. Choose saturated neighborhoods $U\left(L_{x}\right)$ as in Proposition 1 and choose an open covering $\left\{\operatorname{int}\left(U\left(L_{j}\right)\right) ; j \in J=\mathbb{Z} / 2 m\right\}$ ( $m$ is a natural number, but if $\partial B \cong \mathbf{R}$, then $J=\mathbb{Z}, m=\infty)$ of $\pi^{-1}(\partial B)$ such that 1$) k \equiv j$ or $j \pm 1(\bmod 2 m)$ if $\operatorname{int}\left(U\left(L_{k}\right)\right) \cap \operatorname{int}\left(U\left(L_{j}\right)\right) \neq \emptyset$ and 2) $L_{j}$ is a dihedral leaf if and only if $j$ is odd. The open covering of $B$ in the proof of Theorem 9 is modified as in the following Fig. 5.

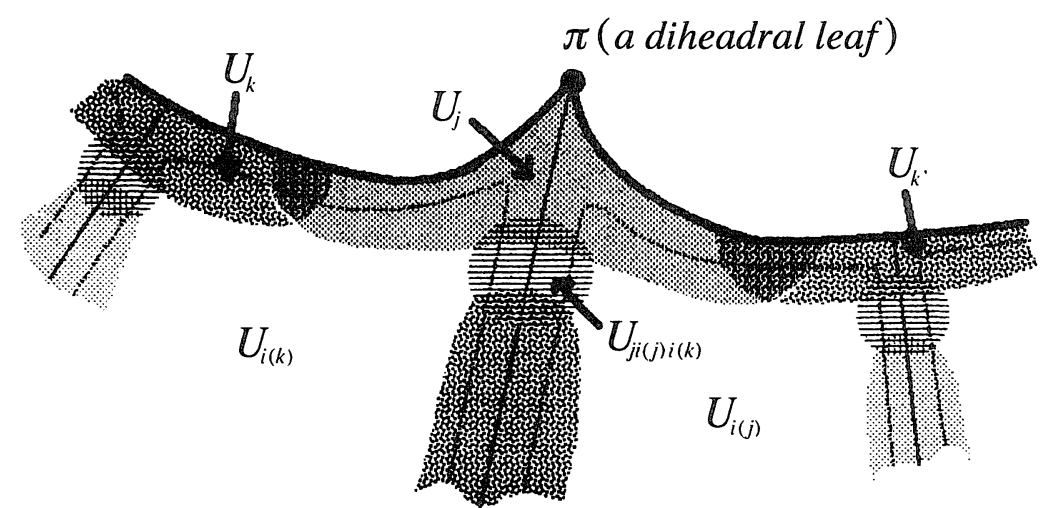

Fig. 5.

First, we perturb $\mathcal{F}$ on $\operatorname{int}\left(U\left(L_{j}\right)\right)$. The holonomy group of a dihedral leaf $L_{j}$ is generated by generators $\tau_{j}, \tau_{j}$, which are generators of adjoining reflection leaves on both sides. Let $p_{j}: L \rightarrow L_{j}$ be the covering maps $(j \in J)$. Then 
Step 1 . In the case where $j$ is odd, take an arbitrary simple closed curve $\delta_{j}$ on $L_{j}$ such that

$$
\left[\delta_{j}\right] \neq 0,\left[\delta_{j}\right] \neq\left[\tau_{j}\right],\left[\delta_{j}\right] \neq\left[\tau_{j^{\prime}}\right],\left\langle\left[\delta_{j}\right],\left[\tau_{j}\right]\right\rangle=\left\langle\left[\delta_{j}\right],\left[\tau_{j^{\prime}}\right]\right\rangle=0 .
$$

This is possible from Lemma 5 . Then we can perturb $\mathcal{F}$ to a foliation which has no compact leaves on $\operatorname{int}\left(U\left(L_{j}\right)\right)$ as in Step 1 of the proof of Theorem 9 .

Step 2. In the case where $j$ is even, we can perturb $\mathcal{F}$ to a foliation which has no compact leaves on $\operatorname{int}\left(U\left(L_{j}\right)\right)$ as in Step 2 of the proof of Theorem 9.

Hence we obtain a new foliation which has no compact leaves on $\cup_{j \in J} \operatorname{int}\left(U\left(L_{j}\right)\right)$. We have to note that on neighborhoods meeting $\operatorname{int}\left(U\left(L_{j}\right)\right)$, at most $n(j)+2$ simple closed curves are induced from $\tau_{j}$ and $\tau_{j^{\prime}}$ in each dihedral leaf, but we can perturb it in the same way as in the proof of Theorem 3 since the genus of the generic leaf $\geq n(j)+1$ and $2\{n(j)+1\}-2 \geq n(j)+2$, where $n(j)$ is the order of the holonomy group of $L_{j}$. This completes the proof.

D) The general case.

Combining Theorems 3, 7, 8, 9 and 10, we have the following.

Theorem 11. All compact Hausdorff $C^{r}$-foliations of $C^{r}$-4-manifolds by hyperbolic surfaces are $C^{r}$-unstable $(1 \leq r \leq \infty)$.

$M$ need not be compact, but if $M$ is compact, then codimension two compact foliations of $M$ are always Hausdorff by the results of Epstein[4], Edwards, Millett and Sullivan[3]. Thus we have the following.

Corollary 12. All compact $C^{r}$-foliations of compact $C^{r}-4$-manifolds by hyperbolic surfaces are $C^{r}$-unstable $(1 \leq r \leq \infty)$.

\section{References}

[1] Bonatti, C., Difféomorphismes commutants des surfaces et stabilité des fibrations en tores, Topology, 29 (1990), 101-126.

[2] Bonatti, C. and Firmo, S., Feuilles compactes d'un feuilletage générique en codimension un, Ann. Sc. Norm. Sup., 27 (1994), 407-462.

[3] Edwards, R., Millett, K. and Sullivan, D., Foliation with all leaves compact, Topology, 16 (1977), 13-32.

[4] Epstein, D. B. A., Periodic flows on three-manifolds, Ann. of Math., 95 (1976), 68-82.

[5] $\longrightarrow$, Foliations with all leaves compact, Ann. Inst. Fourier, Grenoble, 26 (1976), $265-282$.

[6] _ A topology for the space of foliations, Geometry and Topology, Lecture Notes in Math., 597 (1976), 132-150.

[7] Fukui, K., Perturbations of compact foliations, Proc. Japan Acad., 58 (1982), 341-344. 
[8] , Stability of foliations of 3-manifolds by circles, J. Math. Soc. Japan, 39 (1987), 117-126.

[9] $ـ$ Stability and instability of certain foliations of 4-manifolds by closed orientable surfaces, Publ. RIMS, Kyoto Univ., 22 (1986), 1155-1171.

[10] Fuller, F., An index of fixed point type for periodic orbits, Amer. J. Math., 89 (1967), 133-148.

[11] Hirsch, M., Stability of compact leaves of foliations, Dynamical Systems, Acad. Press, (1971), 135-155.

[12] Langevin, R. and Rosenberg, H., On stability of compact leaves and fibrations, Topology, 16 (1977), 107-112.

[13] , Integral perturbations of fibrations and a theorem of Seifert, Differential topology, foliations and Gelfand-Fuks cohomology, Lecture Notes in Math., 652 (1978), 122127.

[14] Plante, J., Stability of codimension one foliations by compact leaves, Topology, 22 (1983), 173-177.

[15] Reeb, G., Sur certaines propriétés topologiques des variétés feuilletées, Act. Sc. et Ind., 1183, Herman, Paris, 1952.

[16] Satake, I., The Gauss-Bonnet theorem for V-manifolds, J. Math. Soc. Japan, 9-4 (1957), 464-492.

[17] Seifert, H., Closed integral curves in 3-spaces and isotopic two dimensional deformations, Proc. A. M. S., 1 (1950), 287-301.

[18] Stowe, D., The stationary set of a group action, Proc. A. M. S., 79 (1980), 139-146.

[19] Thurston, W., A generalization of the Reeb stability theorem, Topology, 13 (1974), $347-352$.

[20] Tsuboi, T., Hyperbolic compact leaves are not $C^{1}$-stable, Geometric study of foliations, World Sci., Singapore, (1994), 437-455. 